\title{
Le syndrome des lymphocytes dénudés
}

Le syndrome des lymphocytes dénudés (SLD) correspond à une absence (ou à une insuffisance) de l'expression des molécules HLA à la surface des lymphocytes T. Il en existe deux formes, selon que le déficit porte sur les molécules de classe I ou de classe II ; dans ces deux maladies, autosomiques récessives, le gène responsable n'est pas génétiquement lié au complexe majeur d'histocompatibilité. L'expression clinique est un déficit immunitaire combiné sévère, probablement lié à une anomalie des processus de réarrangement des gènes codant pour le récepteur spécifique de l'antigène des lymphocytes $T$ et donc à une absence de synthèse d'un récepteur fonctionnel.

\section{Jean-Louis Touraine Professeur à l'université Claude Bernard}

\section{ADRESSE}

J.-L. Touraine : médecine de transplantation Pavillon P, hôpital Édouard-Herriot, 5, place 270

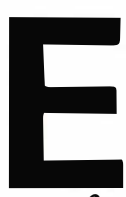
n 1974, alors que nous effectuions l'enquête immuno-génétique à la recherche d'un donneur de moelle osseuse pour un enfant avec déficit immunitaire combiné, nous avons identifié une nouvelle forme de déficit immunitaire, caractérisée par l'absence d'expression des antigènes HLA sur les cellules d'origine hématopoiétique [1]. En anticipation des variations possibles dans le type et le degré du défaut d'expression des déterminants du complexe majeur d'histocompatibilité $(\mathrm{CMH})$, ainsi que dans l'intensité du déficit immunitaire qui en résulte, nous avions désigné cette affection sous le nom de " syndrome des lymphocytes dénudés" (SLD).

Chez ce premier malade, d'origine algérienne, les molécules HLA A et B de classe I étaient absentes des lymphocytes et des plaquettes, tandis que les molécules HLA de classe II étaient exprimées. Peu de temps après, des chercheurs hollandais observaient également une absence d'expression des antigènes HLA A et $B$ chez deux malades porteurs d'un déficit immunitaire combiné
(Tableau I). Il est remarquable que les auteurs, pourtant non avertis de la description initiale, aient observé des anomalies très comparables : déficit identique en fonction immunitaire et absence semblable d'expression d'antigènes HLA de classe I (principalement sur les lymphocytes $\mathrm{T}$ des malades étudiés). De plus, une réduction partielle d'expression des antigènes HLA DR (de classe II) était suggérée, mais elle était beaucoup moins importante que la réduction des antigènes HLA A et $B$ chez ces deux enfants d'origine turque [2].

Un deuxième enfant, de la première famille algérienne, se présentait alors avec un SLD, à nouveau caractérisé par l'absence des antigènes HLA de classe I et un déficit immunitaire sévère. Après ces quatre enfants, 40 autres malades ont été décrits avec un défaut important d'expression soit des antigènes HLA de classe $I$, soit des antigènes HLA de classe II $[1-10]$. Nous les avons respectivement dénommés SLD de type I et SLD de type II.

Parmi les 44 sujets avec des "lymphocytes dénudés ", quatre personnes ont une réduction par- 
tielle de l'expression des antigènes HLA sans déficit immunitaire significatif, ce qui suggère qu'il existe, dans les conditions physiologiques, un excès d'expression des molécules HLA au-dessus de la nécessité minimum pour le fonctionnement adéquat des cellules du système immunitaire [3]. Les 40 autres malades ont un déficit immunitaire de gravité variable ; il s'agit, dans presque tous les cas, d'un déficit immunitaire combiné dont les manifestations cliniques les plus sérieuses semblent apparaître lorsque les cellules des malades sont complètement dépourvues de toute trace d'antigène HLA de classe I.

Cet article résume les principales connaissances sur le SLD, dans ses aspects cliniques, immunologiques et immunogénétiques. Les anomalies d'expression des antigènes HLA de classe I seront ici décrites plus en détail que celles portant sur la classe II, car un autre article de B. Grospierre et C. Griscelli a récemment évoqué plus spécifiquement le SLD de type II $\left(\mathrm{m} / \mathrm{s} n^{\circ} 1\right.$, vol. 3, p. 19).

\section{Données cliniques}

Chez les 22 malades pour lesquels les informations les plus précises ont été obtenues, il apparaît que l'âge du début de la maladie est le plus souvent très précoce, entre le $1^{\text {er }}$ et le $8^{\text {e }}$ mois de la vie. Dans 11 cas sur 22, l'enfant provenait d'une union consanguine, dans 16 cas il était d'origine nordafricaine ; il s'agissait d'un garçon dans 17 cas et d'une fille dans 5 cas. Les manifestations de début étaient 14 fois une diarrhée, 10 fois une candidose buccale et 8 fois une infection du tractus respiratoire. Comme l'indiquent les chiffres précédents, plusieurs malades avaient diverses infections au moment du diagnostic initial. Les symptômes cliniques qui étaient retrouvés avec la plus grande fréquence tout au long de l'évolution sont : une diarrhée rebelle (19 cas), une absence de

Tableau I

\section{HISTORIQUE DU SYNDROME DES LYMPHOCYTES DÉNUDÉS}

1974 : Découverte du syndrome (absence d'expression cellulaire des antigènes HLA de classe $1+$ déficit immunitaire combiné) chez un nourrisson d'origine algérienne. J.-L. Touraine, H. Bétuel, G. Souillet [1].

1976 : Identification de deux autres cas dans une famille turque (constatations comparables à celles effectuées chez le premier malade bien que la $B_{2}$-microglobuline ait été, dans ces derniers cas, détectée sur les lymphocytes B). R.K.B. Schuurman et J.J. Van Rood [2].

1979 : Cas supplémentaire de SLD dans la famille algérienne initiale. J.-L. Touraine et N. Philippe [11].

1979-82 : Onze nouveaux cas rapportés avec défaut d'expression des antigènes HLA soit de classe I soit de classe II + déficit immunitaire combiné. C. Griscelli, W. Kuis $[9,10]$.

1983 : Identification de deux frères avec des lymphocytes " partiellement dénudés " sans déficit immunitaire détecté. R. Payne et L.M. Young [3].

1984 : Treize malades supplémentaires avec déficit immunitaire et défaut d'expression des antigènes HLA (SLD de type I ou de type II) inclus dans le Registre.

1985 : Dix cas additionnels de SLD et deux sujets avec faible expression d'antigènes HLA sans déficit immunitaire important.

Mars 1986: Un malade avec SLD et un adulte japonais avec défaut d'expression des antigènes HLA de classe I associé à un syndrome infectieux sino-pulmonaire chronique [4].

développement staturo-pondéral (20 cas), une pneumonie interstitielle à cytomégalovirus ou à pneumocystis ( 7 cas), d'autres infections sévères (18 cas), des signes neurologiques (5 cas), une complication de l'administration du vaccin polio vivant (2 cas), une anémie hémolytique autoimmune (1 cas).

Les enfants décédés de leur déficit immunitaire sont morts entre les âges de 7 et 66 mois ; les malades, vivant aujourd'hui même avec cette affection, sont âgés de 8 mois à 12 ans. Quatre sujets, dont les cas de 2 frères rapportés par R. Payne et al. [3], n'avaient pas de déficit immunitaire apparent mais, chez eux, le défaut d'expression des antigènes HLA n'était que partiel. Enfin, récemment, un adulte japonais qui présentait des infections sinopulmonaires à répétition, a été trouvé porteur du SLD [4].

\section{Déficit immunologique}

Le déficit d'immunité à médiation cellulaire comporte de façon constante une extrême réduction, voire même une totale absence, d'hypersensibilité retardée aux antigènes de rappel administrés par voie intradermique. Le nombre total de lymphocytes n'est trouvé en permanence en dessous de $1500 / \mathrm{mm}^{3}$ que dans un cas, le nombre de lymphocytes $T$ est en revanche significativement réduit chez huit des 22 malades. La réponse proliférative aux mitogènes est franchement inférieure à la normale chez deux malades parmi les 22 cas étudiés. La réponse proliférative à une stimulation allogénique est réduite dans quatre cas sur 15 et la lymphocytotoxicité à médiation cellulaire dans trois cas sur 11.

L'immunité humorale, chez les 22 sujets rapportés en détail dans le registre du SLD, est caractérisée par une hypogammaglobulinémie constante. La production d'anticorps après vaccination est pratiquement toujours absente. Les allo-hémagglutinines sont diminuées sept fois sur huit. Le nombre de lymphocytes B est normal 


\section{RÉFÉRENCES}

1. Touraine JL, Bétuel H, Souillet G, Jeune M. Combined immunodeficiency disease associated with absence of cell-surface HLA-A and B antigens. $J$ Pediatr 1978 ; 93 : 47-51.

2. Schuurman RKB, Van Rood JJ, Vossen $\mathrm{JM}$, et al. Failure of lymphocyte membrane HLA-A and B expression in two siblings with combined immunodeficiency. Clin Immunol Immunopathol 1979 ; 14 : 418-34.

3. Payne R, Brodsky FM, Peterlin BM, Young LM. "Bare lymphocyte" without immunodeficiency. Hum Immunol 1983; 6 : 219-27.

4. Sugiyama $Y$, Maeda $H$, Okumura $K$, Takaku F. Progressive sinobronchiectasis associated with the "Bare lymphocyte syndrome * in an adult. Chest $1986 ; 89: 398-401$.

5. Touraine JL, Bétuel $H$, Touraine F. The Bare lymphocyte syndrome. In : Griscelli C, Vossen J, eds. Progress in Immunodeficiency Research and Therapy 1. Amsterdam : Elsevier, 1984: 27-34.

6. Bétuel H, Touraine JL, Souillet G, Jeune M. Absence of cell-membrane HLA antigens in an immunodeficient child. Tissue Antigens $1978 ; 11: 68-70$.

7. Touraine JL, Bétuel $\mathrm{H}$. Immunodeficiency diseases and expression of HLA antigens. Hum Immunol 1981 ; 2 : 147-53.

8. Touraine JL. The "Bare lymphocyte syndrome ": Report on the Registry. Lancet 1981 ; i : $319-21$.

9. Griscelli C, Durandy A, Virelizier JL, et al. Impaired cell to cell interaction in partial combined immunodeficiency with defective synthesis and membrane expression of HLA antigens. In : Touraine JL, Gluckman E, Griscelli C, eds. Bone Marrow Transplantation in Europe 11. Amsterdam : Excepta Medica, 1981 : 194-200.

10. Kuis W, Roord J, Zegers BJM, at al. Clinical and immunological studies in a patient with the "Bare lymphocyte " syndrome. In : Touraine JL, Gluckman E, Griscelli C, eds. Bone Marrow Transplantation in Europe II. Amsterdam : Excepta Medica, 1981 : 201-8.

11. Touraine JL, Bétuel H, Philippe N. The Bare lymphocyte syndrome. Immunological studies before and after bone marrow transplantation. Blut 1980 ; 41 : 198-202.

12. Zinkernagel RM, Callahan GN, Klein J, Dennert G. Cytotoxic T cells learn specificity for self $\mathrm{H}-2$ during differentiation in the chez tous les malades, de même que les diverses fractions du complément.

L'observation immunologique la plus constante et la plus impressionnante est l'absence complète de toute réponse cellulaire ou humorale aux antigènes solubles. La comparaison entre les anomalies immunologiques des malades avec SLD de type I et SLD de type II montre moins de différence que ce que l'on aurait pu attendre d'après les connaissances actuelles sur les fonctions respectives des deux variétés de molécules du $\mathrm{CMH}$. Les nuances suivantes peuvent être suggérées, mais devront être confirmées : déplétion un peu plus profonde en lymphocytes $\mathrm{T}$ dans le sang périphérique et les organes lymphoïdes chez les malades avec SLD de type I ; déficit immunitaire relativement plus modéré chez les sujets avec SLD de type II ; fonctions de coopération réduites dans les deux types, mais sans que cela ne soit associé à des anomalies numériques des lymphocytes $\mathrm{T}$ dans le SLD de type II ; fonctions cytotoxiques des lymphocytes $T$ normales dans le type II et en partie réduites chez certains des malades avec SLD de type I [5].

\section{Déficit}

\section{immuno-génétique}

SLD de type I. Par des tests de cytotoxicité et d'immunofluorescence, il a été montré que les antigènes HLA $A, B$ et $C$ sont absents de la surface des lymphocytes des malades avec SLD de type I. Ces antigènes sont aussi absents de la surface des plaquettes et des autres cellules d'origine hématopoiétique. En revanche, les antigènes HLA sont détectés par absorption dans le sérum des malades ; ils sont également trouvés à la surface des fibroblastes cultivés à partir de la peau $[1,6,7]$. De la même façon, la $\beta_{2}$-microglobuline est présente à un taux normal dans le sérum mais n'est pas trouvée à la surface des lymphocytes. Chez certains malades, la $\boldsymbol{\beta}_{2}$-microglobuline est absente de la membrane cellulaire des lymphocytes $T$ et des lymphocytes $\mathrm{B}$, tandis que chez d'autres malades, elle n'est pas trouvée sur les lymphocytes $T$ en suspension mais est détectée en quantité réduite sur les lymphocytes $B[2,8]$. Les antigènes HLA $A$, $B$ et $C$ ainsi que la $\beta_{2}$-microglobuline sont aussi pratiquement absents de la surface des lymphoblastes $\mathrm{B}$ transformés par le virus d'Epstein-Barr au stade initial du développement de la lignée, mais deviennent progressivement détectables, après quelques semaines de culture, sur un nombre de plus en plus important de cellules [11].

Les antigènes HLA de classe II sont présents, en quantité normale ou diminuée, sur les cellules des malades avec un SLD de type I. Les lymphocytes B de ces'sujets sont capables de stimuler la prolifération de lymphocytes $\mathrm{T}$ allogéniques normaux. D'autres antigènes de surface, d'une nature différente (par exemple les immunoglobulines, les antigènes de différenciation des lymphocytes $T$ ) sont trouvés à la surface des lymphocytes appartenant à l'une ou à l'autre des principales populations lymphocytaires. Le déficit d'expression membranaire dans le SLD apparaît donc relativement spécifique des molécules HLA de classe I. Il s'y associe une anomalie des structures de reconnaissance pour l'antigène à la surface des lymphocytes $T$. Les résultats préliminaires des expériences en cours nous suggèrent que l'absence d'expression des antigènes HLA sur diverses cellules du thymus est peut-être responsable d'importantes anomalies dans le processus normal de " réarrangement " des gènes gamma, bêta et alpha codant pour le récepteur spécifique de l'antigène au niveau des cellules $T$. Si ces résultats sont confirmés, on pourra en conclure que l'acquisition de la capacité à reconnaître les divers antigènes par les lymphocytes $T$, au cours de leur ontogénie dans le thymus, dépend de la présence des molécules HLA.

L'effet in vitro de l'interféron alpha recombinant sur l'expression des antigènes HLA dans le SLD de type I peut être résumé de la façon suivante : dans les formes $\mathrm{m} / \mathrm{s} n^{\circ} 5 \mathrm{vol}$. 3, mai 87 


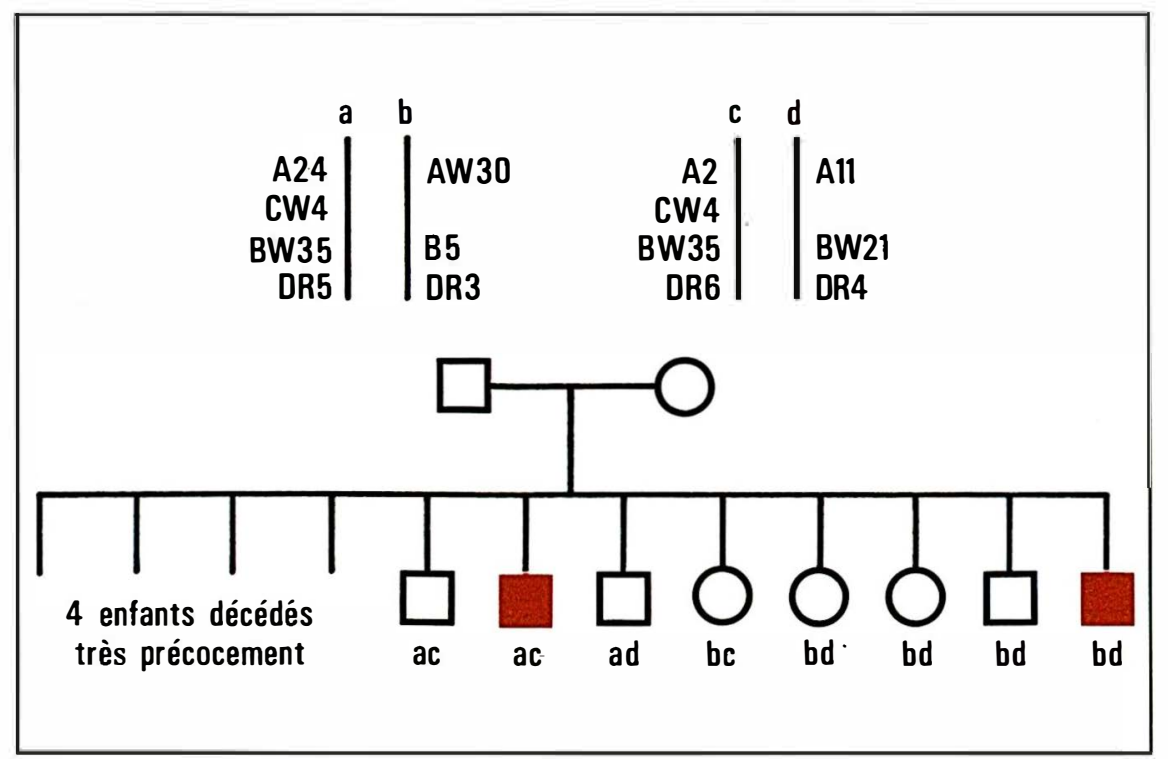

Figure 1. Génotypes HLA déterminés par le Docteur H. Bétuel dans la famille où le syndrome des lymphocytes dénudés a été initialement décrit. Deux garçons ont présenté l'affection (D). Ils avaient hérité de deux chromosomes 6 différents. Chacun d'eux avait en revanche des frères ou sœurs génotypiquement identiques au niveau HLA mais qui ne présentaient pas d'anomalie immunologique, pas de défaut d'expression des antigènes HLA et pas de maladie clinique.

les plus complètes du syndrome, aucune modification significative n'est observée; dans les formes avec déficit partiel d'expression des antigènes HLA de classe I, une augmentation de cette expression peut être constatée par étude cytofluorimétrique [5].

Les analyses familiales suggèrent que le SLD est une affection autosomique récessive due à des gènes différents de ceux de la région du complexe majeur d'histocompatibilité [8]. En effet, dans une famille, les deux malades étaient deux frères ayant hérité de leurs parents de deux chromosomes 6 différents et ayant des génotypes HLA distincts, tandis que chacun d'eux avait des frères ou sœurs "HLA identiques " normaux, sans déficit immunitaire (figure 1). Dans une autre famille où deux frères étaient également atteints, les malades n'avaient pas non plus le même génotype HLA [2]. Le SLD et sa transmission génétique révèlent donc la présence de gène(s) non porté(s) par le chromosome 6 mais contrôlant l'expression de la $B_{2}$-microglobu- line et des antigènes HLA de classe I à la surface des cellules. SLD de type II. A l'opposé des malades avec SLD de type I, ceux présentant le type II ont une expression très fortement réduite, ou nulle, des antigènes HLA de classe II, tandis que les antigènes HLA de classe I sont détectés en quantité normale ou relativement réduite. Une description plus complète du SLD de type II a été présentée dans un article de Barbara Grospierre et Claude Griscelli récemment publié dans médecine/sciences $\left(\mathrm{m} / \mathrm{s} \quad n^{\circ} 1\right.$, vol. 3 , p. 19). Les études cytofluorimétriques des lymphocytes des divers malades ont clairement confirmé qu'il existe deux variétés de SLD : celle avec déficit prédominant d'expression des molécules de classe I et celle avec déficit prédominant d'expression des molécules de classe II. Les études familiales montrent que tous les cas d'une même famille sont soit de type I, soit de type II. Nous avons, pour notre part, observé un nombre comparable de malades du type I et du type II. La présentation clinique n'est pas fondamentalement différente, les cas les plus rapidement graves correspondant cependant aux formes complètes de SLD de type I.

\section{Signification biologique}

Dans le SLD, le défaut d'expression HLA est primitif et n'est pas la conséquence de l'immaturité cellulaire. Malgré les altérations de différenciation cellulaire, les lymphocytes $\mathrm{T}$ sont plus matures dans ce syndrome que dans la plupart des autres déficits immunitaires combinés sévères : présence d'antigènes de différenciation reconnus par les anticorps monoclonaux, réponse proliférative partielle ou sub-normale aux mitogènes et aux cellules allogéniques. Il est bien connu que l'expression HLA est acquise à un stade précoce de l'ontogénie lymphocytaire normale. L'anomalie de développement immunitaire peut donc être considérée comme la conséquence (et non la cause) du déficit immuno-génétique. Deux types de liens peuvent être envisagés entre les deux déficits, lymphocytaire et immunogénétique : (a) déficit primitif d'expression HLA provoquant le déficit immunitaire combiné ; (b) déficit primitif d'une molécule non encore identifiée aboutissant à deux conséquences simultanées : l'absence d'insertion dans la membrane cellulaire de la $B_{2}$-microglobuline et des antigènes HLA d'une part, le déficit immunitaire d'autre part.

Les méthodes de la biologie moléculaire appliquées à l'étude de cette maladie tendent à démontrer que les anomalies de fonction lymphocytaire sont bien la conséquence de l'absence des molécules HLA. Cette absence conduit à l'anomalie des fonctions effectrices du lymphocyte $T$ et à l'incapacité pour cette cellule de reconnaître l'antigène. Les résultats les plus récents, qui demandent à être confirmés par de plus vastes études, suggèrent que l'anomalie fondamentale, conséquence du défaut d'expression des 

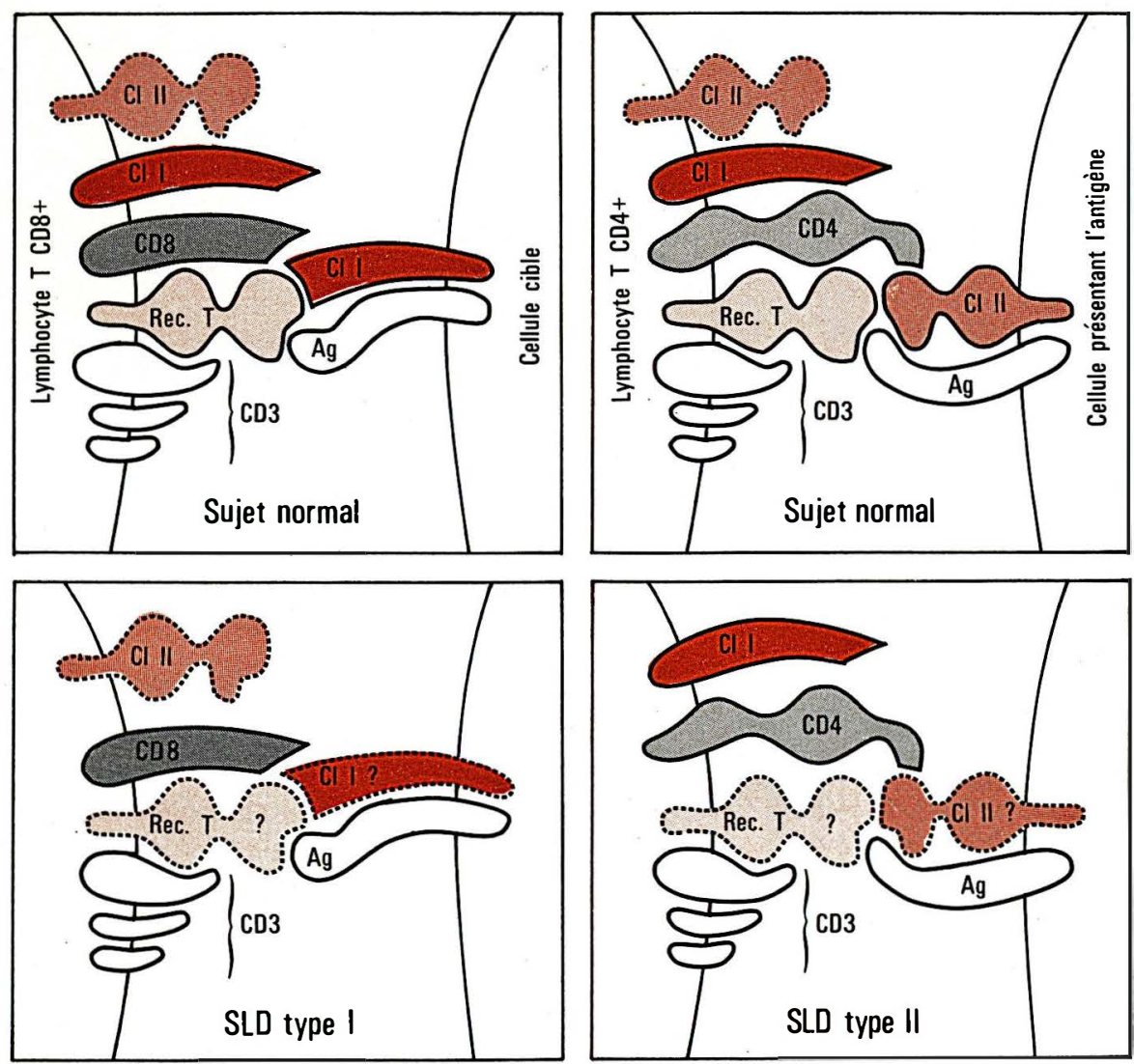

Figure 2. Représentation schématique des antigènes de surface des lymphocytes $T$ qui interviennent dans la reconnaissance de l'antigène. Les anomalies d'expression des antigènes HLA dans les deux variétés de syndromes des lymphocytes dénudés (SLD) perturbent cette fonction fondamentale des lymphocytes. II semble aussi qu'elles puissent être responsables, à un stade plus précoce de l'ontogénie, d'une anomalie d'acquisition du récepteur pour l'antigène, peut-être par anomalie de "réarrangement " des gènes dans les thymocytes. $A g=$ Antigène ; $C D$ 3, 4, 8 = "clusters of différentiation ", c'est-à-dire marqueurs antigéniques de membrane (voir lexique $\mathrm{m} / \mathrm{s}$, nos 2 et 4 , vol. 3, p. 106 et 229) ; Cl l et II : molécules de classe I et II du complexe majeur d'histocompatibilité; Rec. $T$ : récepteur spécifique de l'antigène d̀ la surface du lymphocyte $T$.

antigènes HLA, est l'incapacité d'acquisition du récepteur spécifique de l'antigène à la surface des lymphocytes $T$, par anomalie de "réarrangement " des gènes dans ces cellules au moment de leur ontogénie intra-thymique (figure 2).

Quoi qu'il en soit, l'existence du syndrome des lymphocytes dénudés confirme l'importance fondamentale des antigènes HLA dans le développement du système immunitaire et dans l'exercice de ses fonctions $[5,8,12]$. En l'absence des molécules HLA de classe I et de classe II, il n'y a pas de mécanisme de défense

organismes de notre environnement. Il en résulte habituellement une affection très sévère, spontanément mortelle au cours de l'enfance ou de l'adolescence. Une correction peut être assurée par la greffe de cellules souches normales. Une étude plus poussée des deux types de ce syndrome indiquera la part respective des molécules de classe I et de classe II du CMH dans le développement lymphocytaire et dans l'acquisition de la capacité à reconnaître l'antigène par les cellules $\mathrm{T}$. Enfin, le SLD révèle la présence de gènes, localisés en dehors du chromosome 6 , contrôlant l'expression des antigènes HLA de classe I et de classe II

\section{Summary}

The typical form of the Bare Lymphocyte Syndrome consists of the lack of expression of class I or class II HLA antigens, resulting in combined immunodeficiency. The responsible gene is not borne by chromosome 6 , yet controls the expression of HLA antigens. This syndrome demonstrates the major role of HLA antigens both in $T$ lymphocyte effector functions and in the acquisition of recognition structures for the antigen by $\mathrm{T}$ cells.

\section{REMERCIEMENTS}

Je remercie les Docteurs H. Bétuel, R.K.B. Schuurman, J.J. Van Rood, C. Griscelli, F. Touraine, G. Souillet, G. Lenoir, N. Philippe, H. Renaud, W. Kuss et R.E. Ballieux pour les études réalisées afin d'améliorer la compréhension du SLD. Je remercie également l'Organisation Mondiale de la Santé d'avoir bien voulu supporter l'établissement et le maintien du Registre.

\section{TIRÉS À PART}

J.-L. Touraine : médecine de transplantation, Pavillon P, hôpital Édouard-Herriot, 5, place d'Arsonval, 69437 Lyon Cedex 08. 